\title{
An Intelligent Handoff System for Wireless Cellular Networks Using Fuzzy Logic and Random Walk Model
}

\author{
Leonard Barolli ${ }^{\dagger}$, Fatos Xhafa ${ }^{\ddagger}$, Arjan Durresi ${ }^{\dagger \ddagger}$, Akio Koyama ${ }^{\ddagger \dagger}$, Makoto Takizawa ${ }^{\ddagger \ddagger}$ \\ ${ }^{\dagger}$ Department of Information and Communication Engineering \\ Fukuoka Institute of Technology (FIT) \\ 3-30-1 Wajiro-Higashi, Higashi-Ku, Fukuoka 811-0295, Japan \\ E-mail: barolli@fit.ac.jp \\ ${ }^{\ddagger}$ Department of Languages and Informatics Systems, Polytechnic University of Catalonia \\ Jordi Girona 1-3, 08034 Barcelona, Spain \\ E-mail: fatos@1si.upc.edu \\ $\dagger \ddagger$ Department of Computer and Information Science \\ Indiana University Perdue University Indianapolis \\ 723 W. Michigan Street SL 280, Indianapolis, IN 46202, USA \\ E-mail: durresi@cs.iupui.edu \\ $\ddagger_{\dagger}$ Department of Informatics, Yamagata University \\ 4-3-16 Jonan, Yonezawa 992-8510, Yamagata, Japan \\ E-mail: akoyama@eie.yz.yamagata-u.ac.jp \\ ${ }_{\ddagger}$ Department of Computers and System Engineering, Tokyo Denki University \\ Ishizaka, Hatoyama, Saitama 350-0394, Japan \\ E-mail: taki@takilab.k.dendai.ac.jp
}

\begin{abstract}
In mobile cellular systems the handover is a very important process to maintain the desired Quality of Service (QoS). Many handover algorithms are proposed in the literature. However, to make a better handover and keep the $Q o S$ in wireless networks is very difficult. In this paper, by using random walk model and fuzzy theory we propose a new handover system. The proposed system uses 3 parameters for handoff decision: signal strength from the present Base Station (BS), signal strength from the neighbor BS, and the distance between Mobile Station (MS) and $B S$. The performance evaluation via simulations shows that proposed system can avoid ping-pong effect and has a good handover decision.
\end{abstract}

\section{Introduction}

During the last few years wireless multimedia networks have been a very active research area $[1,2]$. The QoS support for future wireless networks is a very important problem. To guarantee the QoS, a good handover strategy is needed in order to balance the call blocking and call dropping for providing the required QoS $[3,4]$. In the future, the wireless networks will adopt a micro/pico cellular architecture. However, smaller cell size naturally increases the number of handoffs a Mobile Station (MS) is expected to make $[5,6]$.

Many metrics have been used to support handover decisions, including Received Signal Strength (RSS), Signal to Interference Ratio (SIR), distance between the mobile and $\mathrm{BS}$, traffic load, and mobile velocity, where RSS is the most commonly used one. The conventional handover decision compares the RSS from the serving BS with that from one of the target BSs, using a constant handover threshold value (handover margin). The selection of this margin is crucial to handover performance. If the margin is too small, numerous unnecessary handovers may be processed. Conversely, the QoS could be low and calls could be dropped if the margin is too large. The fluctuations of signal strength associated with shadow fading cause the ping-pong effect [7].

Recently, many investigations have addressed handover algorithms for cellular communication systems. However, it is essentially complex to make handover decision considering multiple criteria. Sometimes, the trade-off of some criteria should be considered. Therefore, heuristic approaches based on Neural Networks (NN), Genetic Algorithms (GA) 
and Fuzzy Logic (FL) can prove to be efficient for wireless networks $[8,9,10,11]$. In [10], a multi-criteria handover algorithm for next generation tactical communication systems is introduced. The handover metrics are: RSS from current and candidate base transceivers, ratio of used soft capacity to the total soft capacity of base transceivers, the relative directions and speeds of the base transceivers and the mobile node. In [11], a handover algorithm is proposed to support vertical handover between heterogeneous networks. This is achieved by incorporating the mobile IP principles in combination with FL concepts utilizing different handover parameters.

In this paper, in different from other works we use Random Walk (RW) model and FL to design a new handover system, which is able to avoid ping-pong effect and has a good handover decision. The structure of this paper is as follows. In Section 2, we present the handover decision problem. In Section 3, we give a brief introduction of RW model. In Section 4, we introduce the proposed system. In Section 5, we discuss the simulation results. Finally, some conclusions are given in Section 6.

\section{Handover Decision Problem}

Handoffs which are consistently both accurate and timely can result in higher capacity and better overall link quality than what is available with today systems $[12,13]$. Now with increasing demands for more system capacity, there is a trend toward smaller cells, also known as microcells. Handoffs are more critical in systems with smaller cells, because for a given average user speed, handoff rates tend to be inversely proportional to cell size [5]. The main objectives of handover are link quality maintenance, interference reduction and keeping the number of handoffs low. Also, a handover algorithm should initiate a handoff if and only if the handoff is necessary. The accuracy of a handover algorithm is based on how the algorithm initiates the handover process. The timing of the handoff initiation is also important. There can be deleterious effects on link quality and interference if the initiation is too early or too late.

Because of large-scale and small-scale fades are frequently encountered in mobile environment, it is very difficult for handover algorithm to make an accurate and timely decision. Handover algorithms operating in real time have to make decisions without the luxury of repeated uncorrelated measurements, or of future signal strength information. It should be noted that some of handover criteria information can be inherently imprecise, or the precise information is difficult to obtain. For this reason, we propose a FL-based approach, which can operate with imprecision data and can model nonlinear functions with arbitrary complexity.

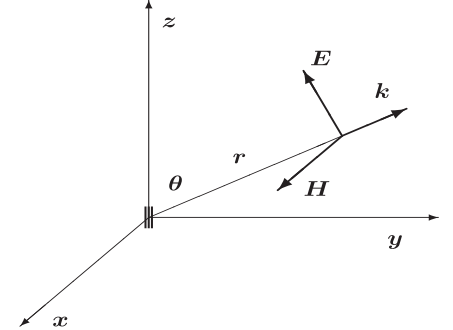

Figure 1. Dipole antenna.

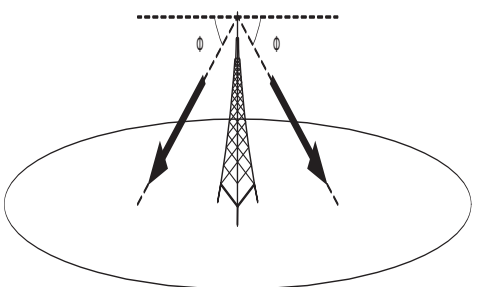

Figure 2. Beam tilting.

\section{RW Model}

We use the MC method for realizing RW model. We consider a 2-dimensional field. The initial position is considered as a origin point and we decided based on MC method the moving pattern for each walk. If we consider $n$ user movements and the angle $\theta$ and distance $d$ for each walk are generated by general or Gaussian distribution, when the movement changes in $x$ and $y$ directions are $\Delta x$ and $\Delta y$, respectively, then we have the following relations.

$$
\begin{gathered}
\Delta x_{n}=d_{n} \cos \theta_{n}, \quad \Delta y_{n}=d_{n} \sin \theta_{n} \\
x_{n+1}=x_{n}+\Delta x_{n}, \quad y_{n+1}=y_{n}+\Delta y_{n}
\end{gathered}
$$

The Base Station (BS) position can be expressed by Cartesian coordinates. By converting Cartesian coordinates to polar ones, we can calculate the angle $\theta$.

We consider that in the cellular system each cell has a hexagonal shape and the BS is located in the center of the cell. The angle $\theta$ between Dipole Antenna (DA) and vector $\boldsymbol{r}$ is $D(\theta)=\sin \theta$. If we consider the transmission power as $W$, the antenna radiation intensity can be calculated as follows:

$$
\boldsymbol{E}=\sqrt{45 W} \sin \theta \frac{e^{-j \kappa r}}{r^{n}} \boldsymbol{u}_{\mathbf{0}}
$$

where, the DA gain is $G=1.5$ and $\boldsymbol{u}_{0}$ is the unit vector that shows DA direction. In Fig. $1 \boldsymbol{u}_{\mathbf{0}}$ is in $Z$ direction.

In Eq.(3), when $\theta=90^{\circ}$, the $E$ value will be maximal in horizontal direction. However, in real situations, the direction of antenna is set up as shown in Fig. 2 in order to cover better the cell area. If we consider the beam tilting angle and the distance, the $E$ can be calculated by the following equation.

$$
\boldsymbol{E}=\sqrt{45 W} \sin (\theta-\phi) \frac{e^{-j \kappa r}}{r^{n}} \boldsymbol{u}_{\mathbf{0}}
$$




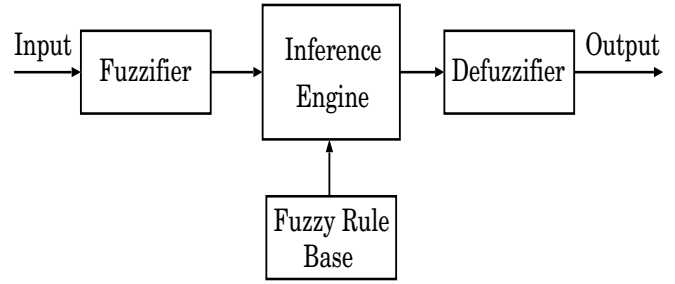

Figure 3. FLC structure.
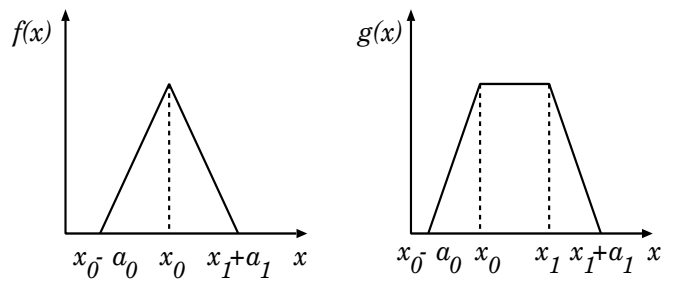

Figure 4. Membership function shapes.

\section{Proposed System Model}

The Fuzzy Logic Controller (FLC) is the main part of the proposed system and its basic elements are shown in Fig. 3. They are the fuzzifier, inference engine, Fuzzy Rule Base (FRB) and defuzzifier. As shown in Fig. 4, as membership functions we use triangular and trapezoidal membership functions because they are suitable for real-time operation [14].

In Fig. $4, x_{0}$ in $f($.$) is the center of triangular function;$ $x_{0}\left(x_{1}\right)$ in $g($.$) is the left (right) edge of trapezoidal func-$ tion; and $a_{0}\left(a_{1}\right)$ is the left (right) width of the triangular or trapezoidal function.

Let us consider the time that a MS is in a cell. In our system, we consider hexagonal cell shape, but for simple calculation let consider a circular cell shape. The distance that a MS moves in a cell can be expressed as follows:

$$
D=2 \sqrt{\left(r^{2}+x^{2}\right)}
$$

where, $0 \leq x<r$.

If the speed of the MS will be $V$, the time that MS is in a cell is:

$$
T_{D}=\frac{D}{V}=\frac{2 \sqrt{\left(r^{2}+x^{2}\right)}}{V} .
$$

The average distance that a MS is in a cell can be calculated by Eq. (7).

$$
\bar{D}=\frac{4 \int_{0}^{r} \sqrt{\left(r^{2}+x^{2}\right)} d x}{2 r}=\frac{\pi r}{2}
$$

From this, the average $T_{D}$ time will be:

$$
\overline{T_{D}}=\frac{\bar{D}}{V}=\frac{\pi r}{2 V} .
$$

The proposed fuzzy model is shown in Fig. 5. We have built 3 fuzzy based systems but for the sake of space in this paper will will present only one. The Node_B shows the

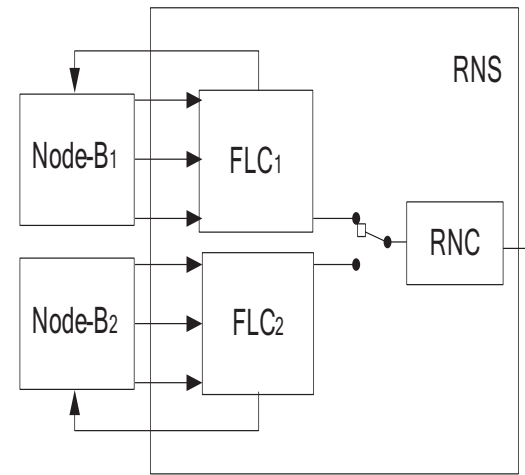

Figure 5. System model.
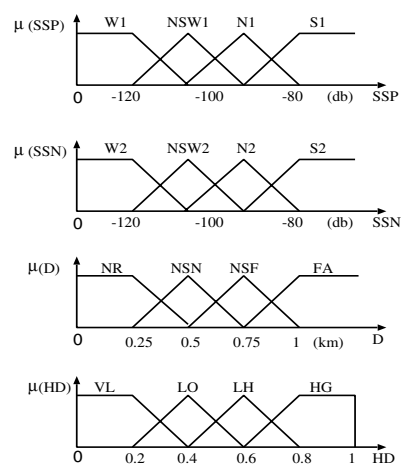

Figure 6. Membership functions.

wireless transmitter and receiver of BS and RNS indicates Radio Network System.

The input parameters for FLC are: Signal Strength from the Present BS (SSP), Signal Strength from the Neighbor BS $(S S N)$, and the distance of MS from BS $(D)$, while the output linguistic parameter is Handover Decision $(H D)$. The term sets of $S S P, S S N$ and $D$ are defined respectively as:

$$
\begin{aligned}
T(S S P) & =\{\text { Weak, Not So Weak, Normal, Strong }\} \\
& =\{W 1, N S W 1, N 1, S 1\} ; \\
T(S S N) & =\{\text { Weak,NotSoWeak, Normal, Strong }\} \\
& =\{W 2, N S W 2, N 2, S 2\} ; \\
T(D) & =\{\text { Near, Not So Near, Not So Far, Far }\} \\
& =\{N R, N S N, N S F, F A\} .
\end{aligned}
$$

The output linguistic parameter $T(H D)$ is defined as $\{$ Very Low, Low, Little High,High $\}=$ $\{V L, L O, L H, H G\}$.

The membership functions of FLC are shown in Fig. 6. The FRB forms a fuzzy set of dimensions $|T(S S P)| \times$ $|T(S S N)| \times|T(D)|$, where $|T(x)|$ is the number of terms on $T(x)$. The FRB is shown in Table 1 and has 64 rules. The control rules have the following form: IF "conditions" THEN "control action". 
Table 1. FRB.

\begin{tabular}{|c|c|c|c|c|c|c|c|c|c|}
\hline Rules & SSP & SSN & $\mathrm{D}$ & HD & Rules & SSP & SSN & $\mathrm{D}$ & HD \\
\hline 1 & W1 & W2 & NR & $\begin{array}{l}\text { VL } \\
\end{array}$ & 33 & N1 & W2 & NR & $\overline{\mathrm{VL}}$ \\
\hline 2 & W1 & W2 & NSN & $\begin{array}{l}\mathrm{VL} \\
\end{array}$ & 34 & N1 & W2 & NSN & $\overline{\mathrm{VL}}$ \\
\hline 3 & $\begin{array}{l}\text { W1 } \\
\end{array}$ & W2 & NSF & VL & 35 & N1 & W2 & NSF & $\mathrm{LO}$ \\
\hline 4 & W1 & W2 & FA & LO & 36 & N1 & W2 & $\overline{F A}$ & LO \\
\hline 5 & W1 & NSW2 & NR & LO & 37 & N1 & NSW2 & NR & LO \\
\hline 6 & W1 & NSW2 & NSN & LO & 38 & N1 & NSW2 & NSN & LO \\
\hline 7 & W1 & NSW2 & NSF & LO & 39 & N1 & NSW2 & NSF & $\overline{\mathrm{LH}}$ \\
\hline 8 & W1 & NSW2 & FA & LH & 40 & N1 & NSW2 & FA & LH \\
\hline 9 & W1 & N2 & NR & LH & 41 & N1 & N2 & NR & LH \\
\hline 10 & $\overline{\text { W1 }}$ & N2 & NSN & $\overline{\mathrm{LH}}$ & 42 & N1 & N2 & $\mathrm{NSN}$ & LH \\
\hline 11 & W1 & N2 & NSF & HG & 43 & N1 & N2 & NSF & LH \\
\hline 12 & W1 & N2 & FA & HG & 44 & N1 & N2 & $\overline{F A}$ & $\mathrm{HG}$ \\
\hline 13 & W1 & S2 & NR & LH & 45 & N1 & S2 & NR & LH \\
\hline 14 & W1 & S2 & NSN & LH & 46 & N1 & S2 & NSN & LH \\
\hline 15 & W1 & S2 & NSF & LH & 47 & N1 & S2 & NSF & LH \\
\hline 16 & W1 & S2 & FA & HG & 48 & N1 & S2 & FA & $\mathrm{HG}$ \\
\hline 17 & NSW1 & W2 & NR & VL & 49 & S1 & W2 & NR & VL \\
\hline 18 & NSW1 & W2 & NSN & VL & 50 & S1 & W2 & NSN & $\mathrm{VL}$ \\
\hline 19 & NSW1 & W2 & NSF & $\overline{L O}$ & 51 & S1 & W2 & NSF & VL \\
\hline 20 & NSW1 & W2 & FA & LO & 52 & S1 & W2 & $\overline{F A}$ & VL \\
\hline 21 & NSW1 & NSW2 & NR & LO & 53 & S1 & NSW2 & NR & $\mathrm{VL}$ \\
\hline 22 & NSW1 & NSW2 & NSN & LO & 54 & S1 & NSW2 & NSN & VL \\
\hline 23 & NSW1 & NSW2 & NSF & $\mathrm{LH}$ & 55 & S1 & NSW2 & NSF & VL \\
\hline 24 & NSW1 & NSW2 & FA & LH & 56 & S1 & NSW2 & $\overline{F A}$ & LO \\
\hline 25 & NSW1 & N2 & NR & $\overline{\mathrm{LH}}$ & 57 & S1 & N2 & NR & LO \\
\hline 26 & NSW1 & N2 & NSN & $\overline{\mathrm{LH}}$ & 58 & S1 & N2 & NSN & $\mathrm{LO}$ \\
\hline 27 & NSW1 & N2 & NSF & $\overline{\mathrm{LH}}$ & 59 & S1 & N2 & NSF & LO \\
\hline 28 & NSW1 & N2 & FA & HG & 60 & S1 & N2 & FA & LH \\
\hline 29 & NSW1 & S2 & NR & LH & 61 & S1 & S2 & NR & LO \\
\hline 30 & NSW1 & S2 & NSN & LH & 62 & S1 & S2 & NSN & LO \\
\hline 31 & NSW1 & S2 & NSF & LH & 63 & S1 & S2 & NSF & LH \\
\hline 32 & NSW1 & S2 & $\overline{F A}$ & $\overline{\mathrm{HG}}$ & 64 & S1 & S2 & $\overline{F A}$ & LH \\
\hline
\end{tabular}

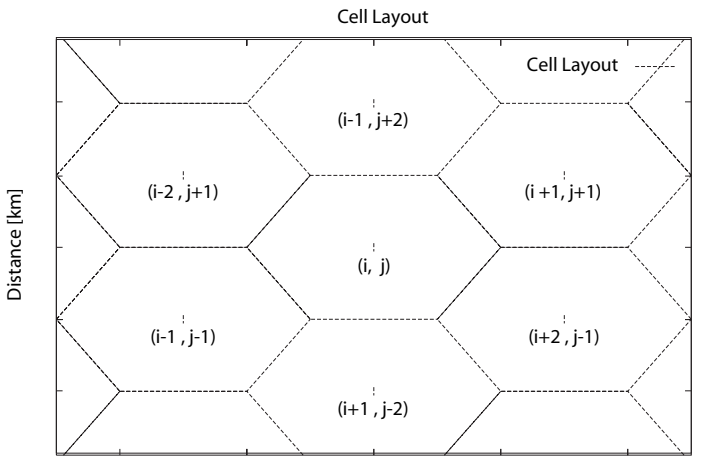

Distance $[\mathrm{km}]$

Figure 7. Cell layout.

\section{Simulation Results}

\subsection{RW model results}

The cell shape is hexagonal and the coordinates of BSs are indicated as shown in Fig. 7. In Fig. 8 is shown the power distribution for one transmission antenna when the BS is located in the center of the cell, the transmission antenna power is $10 \mathrm{~W}$, and cell radius is $2 \mathrm{~km}$. In Table 2 are shown the simulation parameters.

In Fig. 9 is showing the walking pattern for a MS when $i$ seed $=100$ and $n$ walk $=5$, while in Fig. 10 for iseed $=200$ and nwalk $=10$. In Fig. 9, the MS moves in the cells: $(0,0) \rightarrow(2,-1) \rightarrow(0,0) \rightarrow(1,-2)$, while in Fig. 10
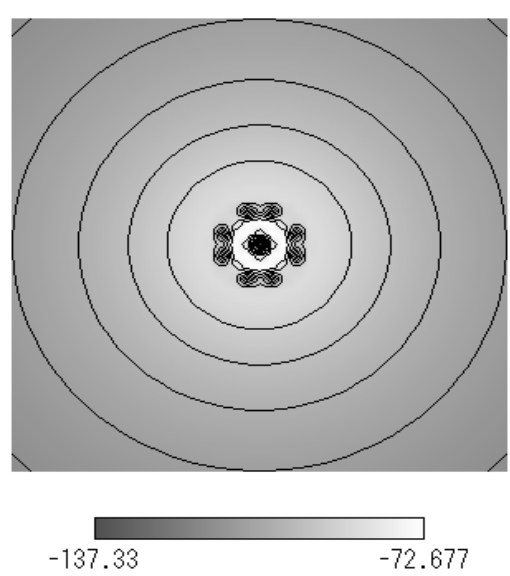

Figure 8. Power distribution for one antenna.

in the cells: $(0,0) \rightarrow(-1,2) \rightarrow(-2,1) \rightarrow(-1,2)$. In Fig. 9, the ping-pong effect happens, because the MS is moving in the cells boundary. While in Fig. 10, the handover process is necessary.

In Fig. 11 is showing the aggregate received power of MS for the case when (iseed = 200). In Fig. 12, Fig. 13 and Fig. 14 is showing the received power from the $\mathrm{BS}(0,0), \mathrm{BS}(2,-1) ! \$ \mathrm{BS}(1,-2)$, respectively. We have also the results for $($ iseed $=100)$, but for the sake of space will 
Table 2. Simulation parameters.

\begin{tabular}{|c|c|}
\hline Distribution Law & Gaussian Distribution \\
\hline Number of Walks & $5 ! \$ 10$ \\
\hline Random Types & $100 ! \$ 200$ \\
\hline Cell Radius & $1 \mathrm{~km} ! \$ 2 \mathrm{~km}$ \\
\hline Transmission Power & $10 \mathrm{~W} ! \$ 20 \mathrm{~W}$ \\
\hline Frequency & $2000 \mathrm{MHz}$ \\
\hline Transmission Antenna Beam Tilting & $3^{\circ}$ \\
\hline Transmission Antenna Height & $40 \mathrm{~m}$ \\
\hline Receiving Antenna Height & $1.5 \mathrm{~m}$ \\
\hline Average Value for a Walk & $0.6 \mathrm{~km}$ \\
\hline$n$ & 1.1 \\
\hline
\end{tabular}

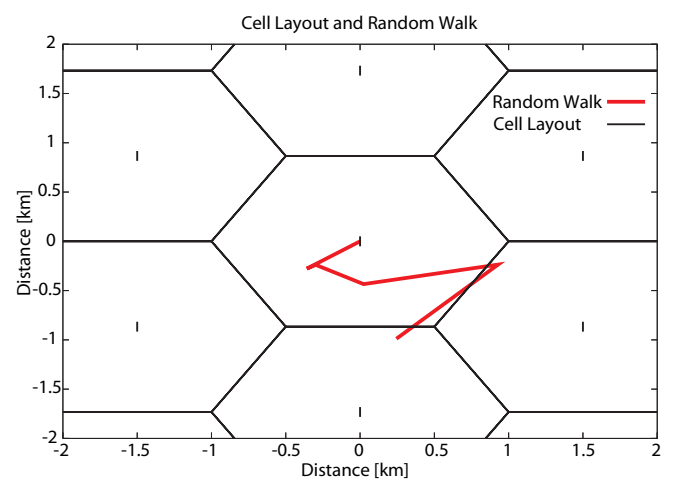

Figure 9. RW pattern for $($ iseed $=100)$.

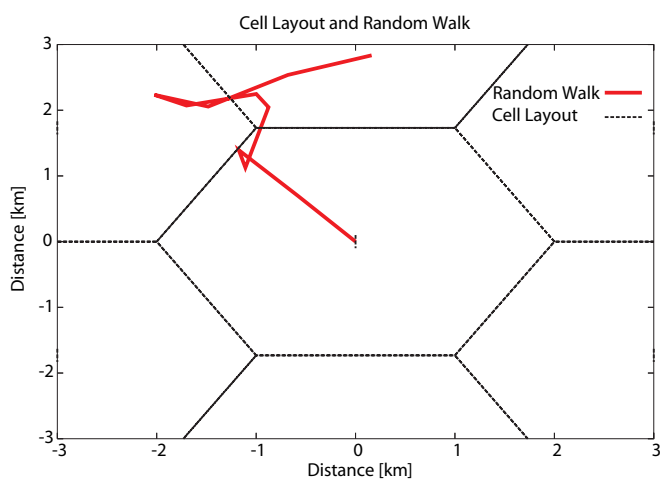

Figure 10. RW pattern for $($ iseed $=200)$.

not show in this paper. As can be seen from Fig. 12, when the MS is going far from the BS the received power is decreased, while when the MS is approaching neighbor BS the received power from these BSs is increased (see Fig. 13 and Fig. 14).

\subsection{Evaluation of Proposed Fuzzy Handover Sys- tem}

For evaluation of the proposed fuzzy-based handover system, we carried out the measurement for 3 points, where the MS is in the boundary of the 3 cells. In Fig. 15 and Fig. 16 are shown the measurement points for $($ iseed $=100$ ) and $(i$ seed $=200)$, respectively. In Fig. 15, the handover should not be carried out, because we will have the pingpong effect, while in Fig. 16 the handover is necessary be-

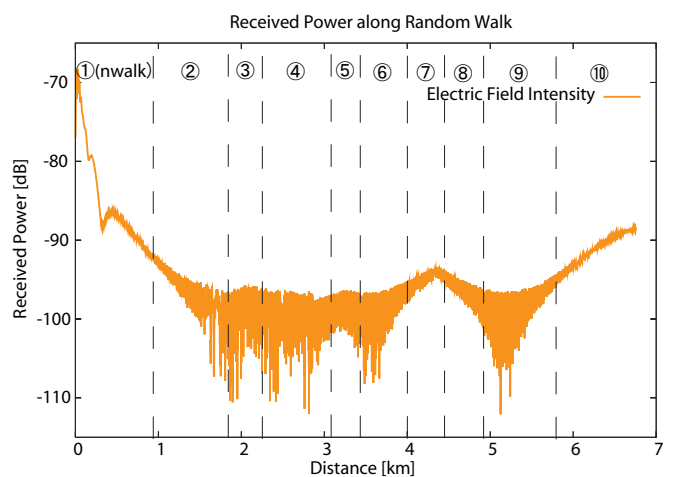

Figure 11. Aggregate received power $($ iseed $=$ 200).

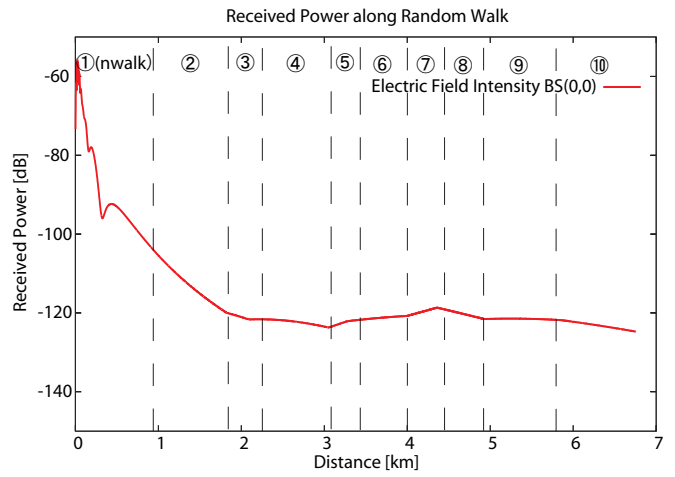

Figure 12. Received power from $\mathrm{BS}(0,0)$ $($ iseed $=200)$.

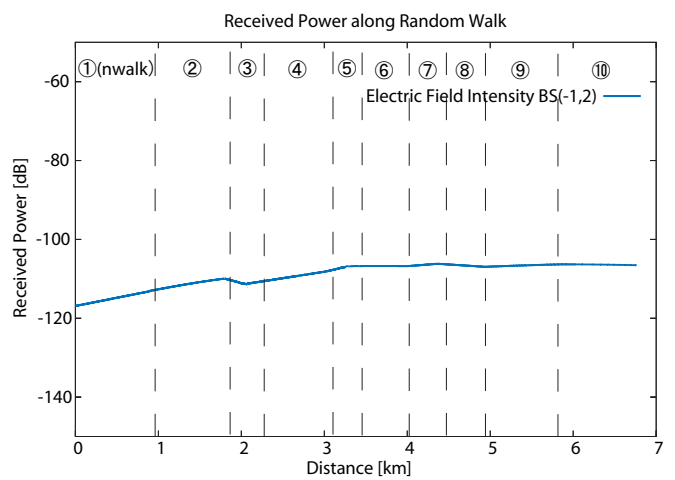

Figure 13. Received power from BS(-1,2) $($ iseed $=200)$.

cause the MS is moving inside the neighbor cells.

In our system, we consider that the handover is carried out when the output value is bigger than 0.7 . We assume that during the RW for each $10 \mathrm{~km} / \mathrm{h}$ the signal strength is decreased $2 \mathrm{db}$. We carry out 10 times simulations and calculate the average values. The simulation results for iseed $=100$ and $i$ seed $=200$ are shown in Table 3 and Table 4, respectively.

In the case when $i$ seed $=100$, the MS moves in the 


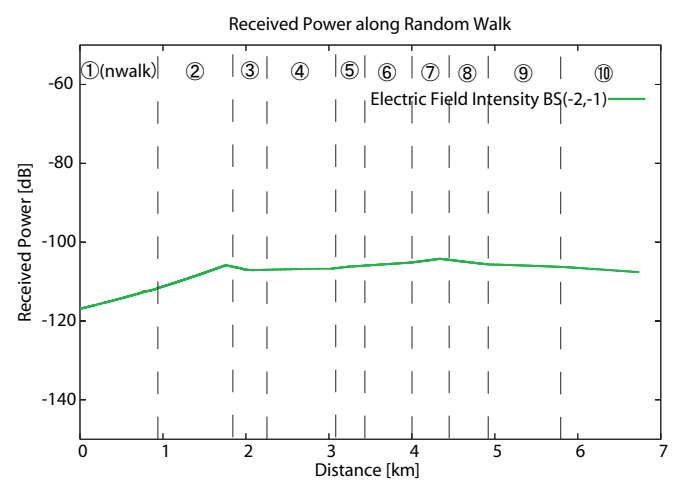

Figure 14. Received power from BS(-2,1) $($ iseed $=100)$.

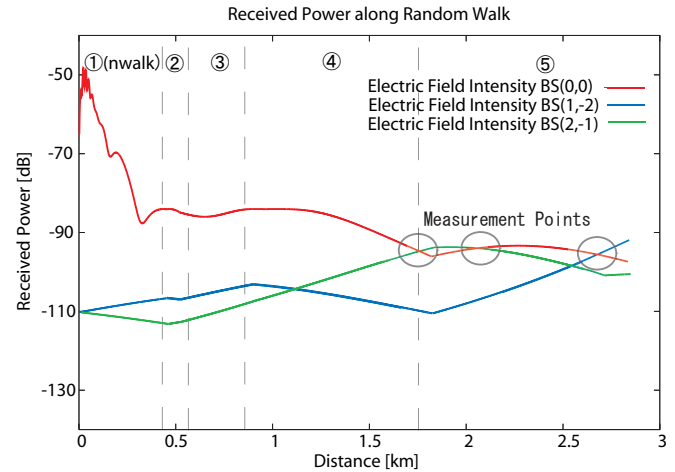

Figure 15. 3 measurement points for $i$ seed $=$ 100.

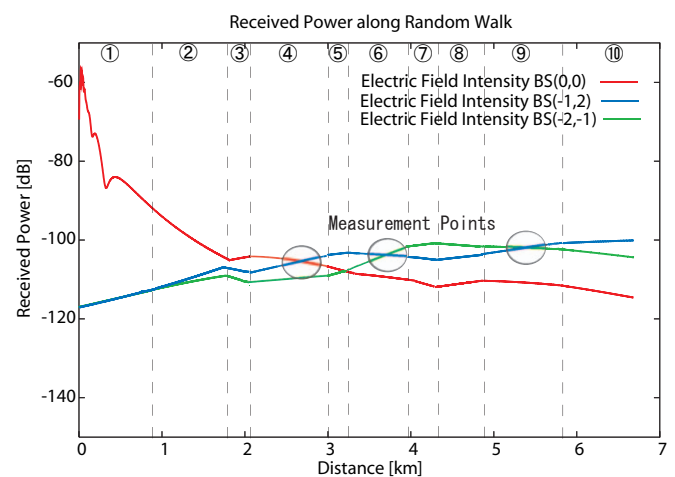

Figure 16. 3 measurement points for $i$ seed $=$ 200.

boundary of cells. Thus if the handover will be carried out, we will have the ping-pong effect. As shown in Table 3, all the average values are smaller than 0.7 , therefore the proposed system can avoid the ping-pong effect.

In the case when iseed $=200$, the MS is moving inside the neighbor cells, so the handover should be carried out 3 times. In the results of Table 4, the proposed system in all cases has done 3 handovers. This shows that the proposed system has a good handover decision.

\section{Conclusions}

In this paper, we proposed a fuzzy based handover system for wireless cellular networks. In different from other works, we used the RW model and FL to design a new handover system. From the simulation results, we conclude that the proposed system is able to avoid ping-pong effect and has a good handover decision.

We have implemented also 2 other fuzzy systems, but for the sake of the space will not show here the simulation results, which will be the topic of other papers.

\section{References}

[1] R. Berezdivin, R. Breining, R. Topp, "Next-Generation Wireless Communication Concepts and Technologies", IEEE Communication Magazine, Vol. 40, No. 3, pp. 108116, 2002.

[2] Y. Guo, H. Chaskar, "Class-Based Quality of Service over Air Interfaces in 4G Mobile Networks", IEEE Communication Magazine, Vol. 40, No. 3, pp. 132-137, 2002.

[3] W. Wang, X. Wang, A.A. Nilsson, 'Energy-Efficient Bandwidth Allocation in Wireless Networks: Algorithms, Analysis, and Simulations", IEEE Transactions on Wireless Communications, Vol. 5, No. 5, pp. 1103-1114, 2006.

[4] Y. Fang, Y. Zhang, "Call Admission Control Schemes and Performance Analysis in Wireless Mobile Networks", IEEE Transactions on Vehicular Technology, Vol. 51, No. 2, pp. 371-382, 2002.

[5] K. D. Wong, D.C. Cox, "A Pattern Recognition System for Handoff Algorithms", IEEE J-SAC, Vol. 18, No. 7, pp. 1301-1312, 2000.

[6] S. Kovvuri, V. Pandey, D. Ghosal, B. Mukherjee, D. Sarkar, "A Call-Admission Control (CAC) Algorithm for Providing Guaranteed QoS in Cellular Networks", International Journal of Wireless Information Networks, Vol. 10, No. 2, pp. 73-85, 2003.

[7] H.P. Lin, R.T. Juang, D.B. Lin, "Validation of an Improved Location-Based Handover Algorithm Using GSM Measurement Data", IEEE Transactions on Mobile Computing, Vol. 4, No. 5, pp. 530-536, 2005.

[8] P. Fiengo, G. Giambene, E. Trentin, "Neural-based Downlink Scheduling Algorithm for Broadband Wireless Networks", Computer Communication, Vol. 30, No. 2, pp. 207218, 2007.

[9] L. Barolli, A. Koyama, T. Suganuma, N. Shiratori, "GAMAN: A GA Based QoS Routing Method for Mobile Ad-hoc Networks", Journal of Interconnection Networks (JOIN), Vol. 4, No. 3, pp. 251-270, 2003.

[10] T. Onel, C. Ersoy, E. Cayirci, "A Fuzzy Inference System for the Handoff Decision Algorithms in the Virtual Cell Layout Base Tactical Communications System", IEEE Military Communications Conference (MILCOM-2002), Vol. 1, pp. 436-441, 2002.

[11] P. M. L. Chan, R. E. Sheriff, Y.F. Hu, P. Conforto, C. Tocci, "Mobility Management Incorporating Fuzzy Logic for a Heterogeneous IP Environment", IEEE Communications Magazine, Vol. 39, No. 12, pp. 42-51, 2001. 
Table 3. Simulation results for iseed $=100$.

\begin{tabular}{|c|c|c|c|c|c|c|}
\hline Measurement Points & \multicolumn{2}{|c|}{ Point 1 } & \multicolumn{2}{c|}{ Point 2 } & \multicolumn{2}{c|}{ Point 3 } \\
\hline \hline \multicolumn{7}{|c|}{ Speed $0 \mathrm{~km} / \mathrm{h}$} \\
\hline Present BS & -93.06 & -94.11 & -92.86 & -92.47 & -94.01 & -95.28 \\
\hline Neighbor BS & -93.36 & -92.49 & -92.77 & -93.98 & -93.99 & -91.28 \\
\hline Distance & 0.8804 & 0.9431 & 0.8684 & 0.8466 & 0.9367 & 1.0183 \\
\hline System Output Value & 0.595 & 0.629 & 0.602 & 0.576 & 0.623 & 0.704 \\
\hline \multicolumn{7}{|c|}{ Speed $10 \mathrm{~km} / \mathrm{h}$} \\
\hline Present BS & -95.06 & -96.11 & -94.86 & -94.47 & -96.01 & -97.28 \\
\hline Neighbor BS & -95.36 & -94.49 & -94.77 & -95.98 & -95.99 & -93.28 \\
\hline Distance & 0.8858 & 0.9431 & 0.8684 & 0.8466 & 0.9367 & 1.0183 \\
\hline System Output Value & 0.598 & 0.649 & 0.600 & 0.578 & 0.623 & 0.728 \\
\hline \multicolumn{7}{|c|}{ Speed 20 km/h } \\
\hline Present BS & -97.06 & -98.11 & -96.86 & -96.47 & -98.01 & -99.28 \\
\hline Neighbor BS & -97.36 & -96.49 & -96.77 & -97.98 & -97.99 & -95.28 \\
\hline Distance & 0.8804 & 0.9431 & 0.8684 & 0.8466 & 0.9367 & 1.0183 \\
\hline System Output Value & 0.568 & 0.621 & 0.572 & 0.538 & 0.590 & 0.696 \\
\hline \multicolumn{7}{|c|}{ Speed $30 \mathrm{~km} / \mathrm{h}$} \\
\hline Present BS & -99.06 & -100.11 & -98.86 & -98.47 & -100.01 & -101.28 \\
\hline Neighbor BS & -99.36 & -98.49 & -98.77 & -99.98 & -99.99 & -97.28 \\
\hline Distance & 0.8804 & 0.9431 & 0.8684 & 0.8466 & 0.9367 & 1.0183 \\
\hline System Output Value & 0.522 & 0.585 & 0.531 & 0.482 & 0.542 & 0.662 \\
\hline \multicolumn{7}{|c|}{ Speed $40 \mathrm{~km} / \mathrm{h}$} \\
\hline Present BS & -101.06 & -102.11 & -100.86 & -100.47 & -102.01 & -103.28 \\
\hline Neighbor BS & -101.36 & -100.49 & -100.77 & -101.98 & -101.99 & -99.28 \\
\hline Distance & 0.8804 & 0.9431 & 0.8684 & 0.8466 & 0.9367 & 1.0183 \\
\hline System Output Value & 0.534 & 0.597 & 0.521 & 0.497 & 0.590 & 0.672 \\
\hline \multicolumn{7}{|c|}{ Speed $50 \mathrm{~km} / \mathrm{h}$} \\
\hline Present BS & -103.06 & -104.11 & -101.86 & -104.47 & -104.01 & -105.28 \\
\hline Neighbor BS & -103.36 & -102.49 & -102.77 & -103.98 & -103.99 & -101.28 \\
\hline Distance & 0.8804 & 0.9431 & 0.8684 & 0.8466 & 0.9367 & 1.0183 \\
\hline System Output Value & 0.576 & 0.625 & 0.566 & 0.549 & 0.600 & 0.668 \\
\hline
\end{tabular}

Table 4. Simulation results for $i$ seed $=200$.

\begin{tabular}{|c|c|c|c|c|c|c|}
\hline Measurement Points & \multicolumn{2}{|c|}{ Point 1} & \multicolumn{2}{|c|}{ Point 2} & \multicolumn{2}{|c|}{ Point 3} \\
\hline \multicolumn{7}{|c|}{ Speed $0 \mathrm{~km} / \mathrm{h}$} \\
\hline Present BS & -105.23 & -108.70 & -104.64 & -107.96 & -103.95 & -111.93 \\
\hline Neighbor BS & -105.55 & -102.07 & -103.52 & -96.763 & -103.85 & -88.422 \\
\hline Distance & 1.9597 & 2.4628 & 1.8367 & 2.3453 & 1.8021 & 3.0449 \\
\hline System Output Value & 0.596 & 0.706 & 0.615 & 0.748 & 0.601 & 0.800 \\
\hline \multicolumn{7}{|c|}{ Speed $10 \mathrm{~km} / \mathrm{h}$} \\
\hline Present BS & -107.23 & -110.70 & -106.64 & -109.96 & -105.95 & -113.93 \\
\hline Neighbor BS & -107.55 & -104.07 & -105.52 & -98.763 & -105.85 & -90.442 \\
\hline Distance & 1.9597 & 2.4628 & 1.8367 & 2.3453 & 1.8021 & 3.0449 \\
\hline System Output Value & 0.595 & 0.715 & 0.616 & 0.799 & 0.601 & 0.800 \\
\hline \multicolumn{7}{|c|}{ Speed $20 \mathrm{~km} / \mathrm{h}$} \\
\hline Present BS & -109.23 & -112.70 & -108.64 & -111.96 & -107.95 & -115.93 \\
\hline Neighbor BS & -109.55 & -106.07 & -107.52 & -100.76 & -107.85 & -92.422 \\
\hline Distance & 1.9597 & 2.4628 & 1.8367 & 2.3453 & 1.8021 & 3.0449 \\
\hline System Output Value & 0.592 & 0.701 & 0.699 & 0.799 & 0.602 & 0.800 \\
\hline \multicolumn{7}{|c|}{ Speed $30 \mathrm{~km} / \mathrm{h}$} \\
\hline Present BS & -111.23 & -114.70 & -110.64 & -113.96 & -109.95 & -117.93 \\
\hline Neighbor BS & -111.55 & -108.07 & -109.52 & -102.76 & -109.85 & -94.422 \\
\hline Distance & 1.9597 & 2.4628 & 1.8367 & 2.3453 & 1.8021 & 3.0449 \\
\hline System Output Value & 0.632 & 0.705 & 0.618 & 0.733 & 0.603 & 0.800 \\
\hline \multicolumn{7}{|c|}{ Speed $40 \mathrm{~km} / \mathrm{h}$} \\
\hline Present BS & -113.23 & -116.70 & -112.64 & -115.96 & -111.95 & -119.93 \\
\hline Neighbor BS & -113.55 & -110.07 & -111.52 & -104.76 & -111.85 & -96.422 \\
\hline Distance & 1.9597 & 2.4628 & 1.8367 & 2.3453 & 1.8021 & 3.0449 \\
\hline System Output Value & 0.602 & 0.711 & 0.660 & 0.711 & 0.647 & 0.800 \\
\hline \multicolumn{7}{|c|}{ Speed $50 \mathrm{~km} / \mathrm{h}$} \\
\hline Present BS & -115.23 & -118.70 & -114.64 & -117.96 & -113.95 & -121.93 \\
\hline Neighbor BS & -115.55 & -112.07 & -113.52 & -106.76 & -113.85 & -98.422 \\
\hline Distance & 1.9597 & 2.4628 & 1.8367 & 2.3453 & 1.8021 & 3.0449 \\
\hline System Output Value & 0.693 & 0.746 & 0.694 & 0.748 & 0.683 & 0.800 \\
\hline
\end{tabular}

[12] S. Mohanty, I.F. Akyildiz, "A Cross-Layer (Layer 2+3) Handoff Management Protocol for Next-Generation Wireless Systems", IEEE Transactions on Mobile Computing, Vol. 5, No. 10, pp. 1347-1360, 2006.

[13] F. Yu, Y. Krishamurthy, "Optimal Joint Session Admission
Control in Integrated WLAN and CDMA Cellular Networks with Vertical Handoff", IEEE Transactions on Mobile Computing, Vol. 6, No. 1, pp. 126-139, 2007.

[14] D. Dubois, H. Prade, R. Yager, (Eds.), "Fuzzy Sets for Intelligent Systems", Morgan Kaufman Publishers, 1993. 